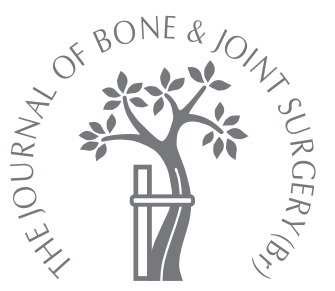

\title{
ANNOTATION The OrthoChina project
}

\author{
M. Zhen-Sheng, \\ Z. Hong-Ju, \\ Y. Tao, \\ R. Gang, \\ D. Guo-Sheng, \\ W. Yong-Hua
}

From Fourth Military

Medical University,

Xi'an, China

\begin{abstract}
M. Zhen-Sheng, MD Associate Professor Department of Orthopaedics Z. Hong-Ju, BSc, Head Nurse Department of Obstetrics and Gynecology

Xijing Hospital, Fourth Military Medical University, 15th Changle West Road, Xi'an, 710032, China.

In. Tao, MD, Orthopaedic Surgeon

Department of Orthopaedics

Pingliang Hospital in

Traditional Chinese Medicine,

Pingliang 744000, Gansu

Province, China.

R. Gang, BSc, Software Engineer

Software Engineering College, $\mathrm{Xi}^{\prime}$ an Jiaotong University, Xi'an 710049, China. licensing subspecialties. Surgeons will have a licence to practise surgery overall, including general surgery, orthopaedic surgery and gynaecology and obstetrics. Outside the major centres most orthopaedic surgeons in China also undertake general surgery in their clinical practice.

Over the past nine years the authors have developed an internet-based website for orthopaedics in China, OrthoChina. ${ }^{1}$ We have evolved a system for identifying the level of expertise of the orthopaedic surgeons who use the site and have created categories of users who are registered to the site, which is based on the presentation and discussion of appropriate cases.
\end{abstract}

D. Guo-Sheng, MD, Orthopaedic Surgeon Rende Hospital, Wuxi 214028, Jiangsu Province, China.

W. Yong-Hua, BSc, Project Manager

Data Bureau of China

Telecommunication Group in Weinan City of Shaanxi

Province, Weinan 714000

China.

Correspondence should be sent to Dr M. Zhen-Sheng; e-mail: mzsheng@fmmu.edu.cn

(C)2007 British Editorial Society of Bone and Joint Surgery doi:10.1302/0301-620X.89B6. $19377 \$ 2.00 \mathrm{~J}$ Bone Joint Surg [Br] 2007;89-B:717-20.

\section{Organisation of the site}

In order to ensure the copyright and safety of the operating system, we selected the free open source coded Redhat Linux AS2 (Redhat Inc. China, Beijing, China) as the operating system, and MySQL (MySQL Inc., Cupertino, California) as the database in conjunction with Tomcat-connector, Apache and Tomcat (The Apache Software Foundation, Forest Hill, Maryland). All these programmes are used freely for the operation of OrthoChina.

OrthoChina acts as a non-profit website only for orthopaedic surgeons. It has been registered with the appropriate National and

\section{Online case discussion may be used for the education of orthopaedic surgeons. The authors developed a website for discussing orthopaedic cases nine years ago and describe its management. It currently has 20000 registered users.}

Interactive online case discussion is a costeffective means of furthering continual medical education. It allows orthopaedic surgeons to follow and take part in discussions about cases and be able to both teach and learn at the same time. However, the different national medical systems make it difficult to determine whether or not the user is an orthopaedic surgeon, especially in the international discussions. In China, we do not have a natural system for
Regional bodies (Beijing Administration Bureau for Industry and Commerce, the Information Safety Agency of Shaanxi Communications Adminstration, and the Internet Administrative Agency of Xi'an Public Security Bureau, Shaanxi) as required by Chinese law.

The webmaster and Chief Editor of OrthoChina is a senior orthopaedic surgeon (MZ-S) with a wide background and experience in orthopaedics. The software and hardware engineering is supported by professional engineers from Software Engineering College, Xi'an Jiaotong University, Xi'an, and the Data Bureau of China Telecommunication Group in Weinan City of Shaanxi Province, China. Their support allows the website to be well managed and academically sound, with a well controlled software environment. The moderators of each forum are qualified orthopaedic surgeons who are experienced in a related subspecialty. They are trained in the management of the system, the regulations and requirements for using the site, and the standard procedures for dealing with the posts. They are required to be supervised by each other to maintain standards. Moderators are elected by an open voting programme. The applicants should be qualified orthopaedic surgeons, with a well-established reputation and enthusiasm to advance the aims of the forum. The voting programme is as follows: apply, voted, elected, trained, internship, on duty under standard election rules and procedures.

Election of moderators. The moderator should be a registered user who is actively interested in taking part in the case discussions and management of the website. There are no limitations regarding age, area, gender or employing authority. Each forum has two to five moderators. The academic moderators are senior 


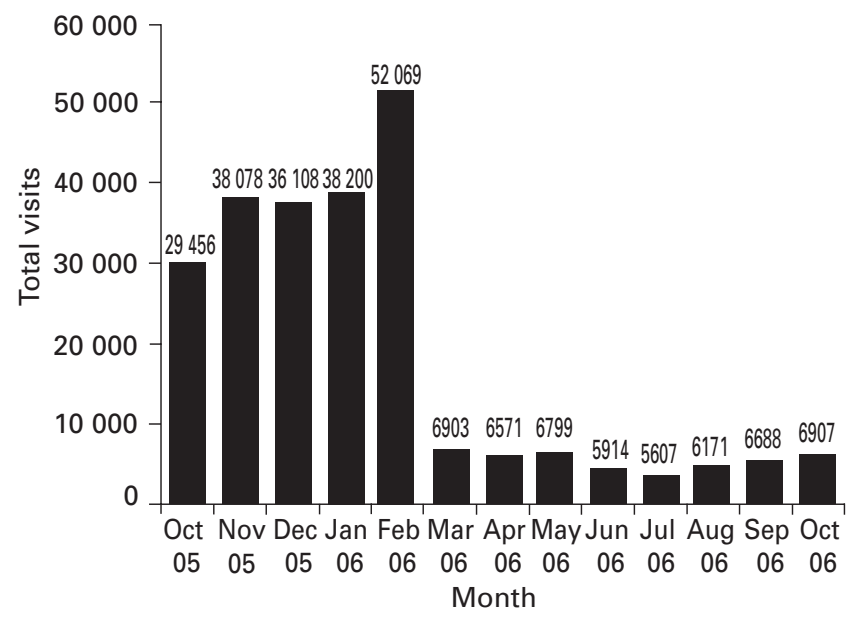

Fig. 1a

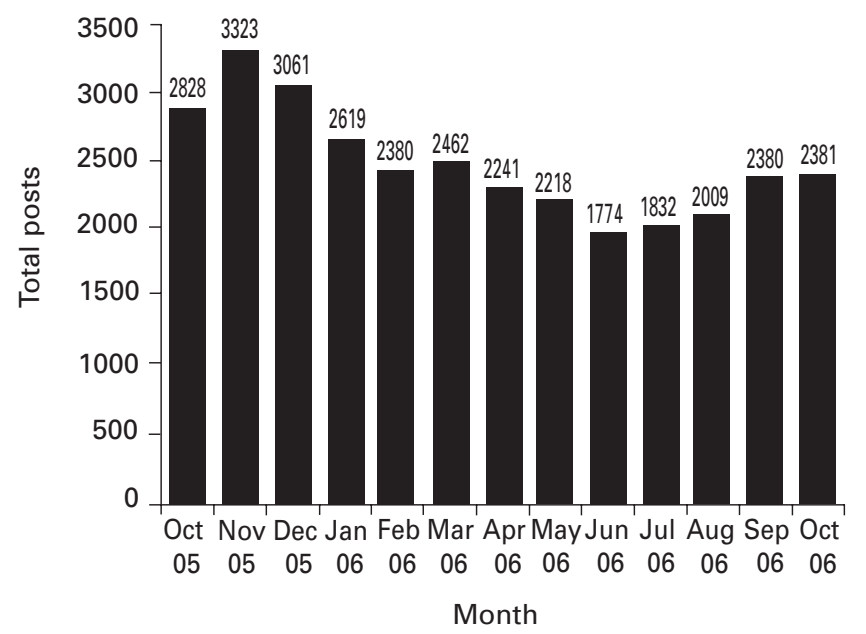

Fig. 1c

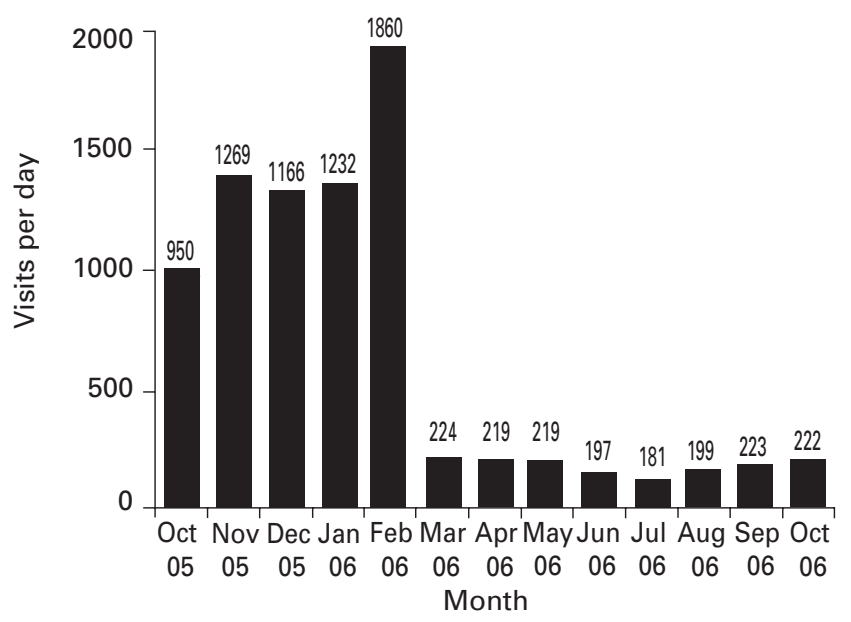

Fig. 1b

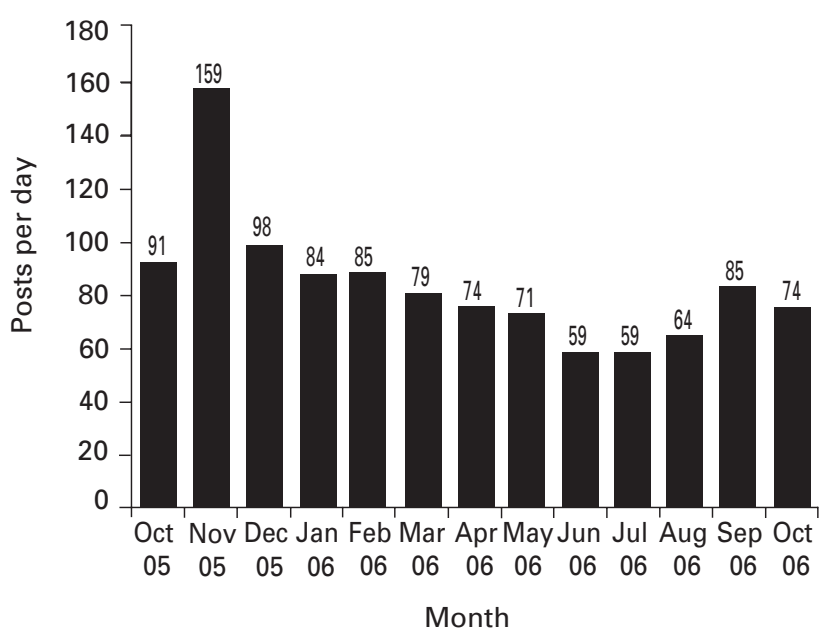

Fig. 1d

Barcharts showing a) monthly total visit statistics (from March 2006 only registered users are included), b) average visits per day (from March 2006 only registered users are included), c) monthly post statistics, including the primary posts and replies, and d) average posts per day, including primary posts and replies.

orthopaedic surgeons who are responsible for the case discussions, giving suggestions for the diagnosis and treatment of the posted cases, and guiding the direction of the discussion. The administrative moderators are junior orthopaedic surgeons who are experienced in managing the forums and are responsible for editing, deleting, and tidying up the forum as appropriate, as well as the quality of the posted cases and the presentation of the images and required information. After applying to be a moderator, the candidate has to show their knowledge of orthopaedics and the ability to manage the forum. They must solicit the support of 20 others over the next seven days. Following this, the applicant will then be elected and trained in managing the forum, and progress to an internship for a further seven days. A further 20 supporting votes are then required to become a moderator. The applicant should obtain the per- mission and agreement of those who are currently moderators in the related forum. If the moderator does not work well and the users are not satisfied, he can be voted off the management team.

All the material presented is viewed, selected and edited by the administrative staff members. The information posted in the forums is monitored by the administrators and moderators.

\section{Management of users of OrthoChina}

Classification of users. The registered users are divided into three groups named Orthopod (orthopaedic surgeon), Other (other medical staff or non-orthopaedic personnel), and Patient (those seeking advice or a second opinion). Unregistered users are called Guests. The Orthopod can view all the information and post in all forums, but the 
Other and Patient groups can only view limited contents and post in limited forums open specifically to them. The Guest category can only view limited contents and cannot post any messages on the site.

Record of registration and logging on. When the user registers his details, the timing and length of his visit and previous visits are recorded automatically on the server and displayed in the administrative pages. These details cannot be changed by the user and are retained for future use.

Establishment of an Orthopod identity. Four procedures are required to confirm the Orthopod identity. The first is a question and answer test. OrthoChina has 2300 single- and multiple-choice questions and answers on a test database. To obtain the identity of Orthopod, the user has to complete ten questions and answers abstracted randomly and automatically from the database, and answer at least seven of the ten correctly. The applicants must present their credentials to show that they are a licensed orthopaedic surgeon, and then be recommended by a collegue. Should the applicant not wish to take the question and answer test, a recommendation from their employer must be provided.

Assessment of use. In order to stimulate the registered users to take an active part in the discussion and to improve the quality of the postings, OrthoChina uses a credit score system. Each user automatically gains one credit point for each primary posting. The administrator and/or moderator can add more to the credit score based on the quality of the post submitted by the user. The administrator and moderator can add to the credit score of a user who gives qualified and valuable comment in case discussion. Each month the users who achieve the top three credit scores will be given additional credits. The credit score is debited when the user downloads or views the orthopaedic videos, PPT slides (files in Microsoft Powerpoint Presentation format; Microsoft Corporation, Redmond, Washington) and electronic orthopaedic textbooks. The users are encouraged to post suitable cases and videos, sharing documents, textbooks and PPT slides. If the primary or reply posting is deleted by the moderator or administrator, the user's score will lose one point. If the user posts unauthorised advertisements or spam after being warned by the moderators, further points will be deducted. Should he continue such practices, his access to OrthoChina will be blocked.

Case presentation. Standard guidelines have been established in order to maintain the quality of presentation of the cases. The contents of the case should be true. The names of patients should not be displayed so as to protect their privacy.

The presentation should include:

1) the gender and age of the patient, their main symptom, the history of the presenting condition, including the time of onset, the development and characteristics of the case, the previous diagnosis, treatment and procedures, the current diagnosis and treatment, the details of examination, etc.,
2) imaging information, which must be as clear and complete as possible. For example, if only anteroposterior and lateral radiographs are provided for a case of lumbar spondylolisthesis, bilateral oblique views must be obtained,

3) the users are encouraged to provide other imaging information, including a video of the physical examination of the patient,

4) details of haematological and bacteriological investigations should be submitted,

5) the author's diagnosis, the purpose of the discussion and the lessons learned by the author should be available,

6) if the patient has undergone surgery, the details should be provided with pathological information if appropriate.

Other management methods. The IP address (internet protocol address for a device on a TCP/IP network) will be displayed when the user logs into OrthoChina and will be displayed in the post. IP address blocking is applied to users who do not confirm with OrthoChina's rules. Keyword filtering is used to filter sensitive non-orthopaedic posts or advertisements.

\section{Use of OrthoChina}

We collected the number of visits from October 2005 to October 2006. From October 2005 to February 2006 Guest visits are included but from March 2006 registered users only are counted (Fig. 1). By 9 November 2006 there were 18975 registered users, of which 5825 had passed OrthoChina's confirmation procedures for orthopaedic surgery.

\section{Discussion}

The work-based case discussion is a most effective educational activity for orthopaedic surgeons worldwide. There are e-mail lists such as Orthogate Orthopod Mailing List, ${ }^{2}$ India Orth Mailing List, ${ }^{3}$ Trauma-List,${ }^{4}$ and Spineconnect forums, ${ }^{5}$ China Spine forums, ${ }^{6}$ E-Radius Forum, ${ }^{7}$ Orthopaedicweblinks forum, ${ }^{8}$ and Ding Xiang Yuan Medical forums. ${ }^{9}$

The OrthoChina project is an open interactive internetbased orthopaedic website for online case discussions, nationally or internationally. Based on their experience of running a forum, the authors have created a system for the management of case discussions comprising its legality, the selection of administrative staff members, the registration of users, confirmation of the identity of orthopaedic surgeons, recording the users' online data, a credit scoring system, an IP filter and a sensitive words filter. Currently, there are over 20000 registered users, 6000 of whom have passed the identity confirmation procedure.

\section{Supplementary Material}

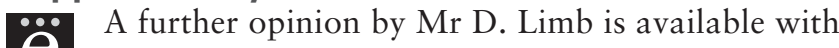
the electronic version of this article on our website at www.jbjs.org.uk 


\section{References}

1. Zhen-Sheng M. OrthoChina, the web on Orthopaedics in China. http:// www.orthochina.org (date last accessed 31 May 2007).

2. No authors listed. ISOST (Internet Society of Orthopaedic Surgery and Trauma) Orthogate Mailing List. http://www.orthogate.org/mailing-lists/orthopod/ (date last accessed 31 May 2007).

3. Parihar M. India Orth Mailing List. http://www.indiaorth.index.html (date las accessed 31 May 2007).

4. No authors listed. Trauma-List http://www.traumalist.html (date last accessed 31 May 2007)
5. No authors listed. Spineconnect http://www.spineconnect.com (date last accessed 31 May 2007).

6. Li-Yang D. China Spine Forum. http://www.jzwk.com (date last accessed 31 May 2007).

7. Nelson DL. The International Distal Radius Fracture Study Group Forum. http:// www.eradius.com/forum-link.htm (date last accessed 31 May 2007).

8. Clough M. The Orthopaedicweblinks Forums. http://www.orthopaedicweblinks.com/forum (date last accessed 31 May 2007).

9. No authors listed. Ding Xiang Yuan Medical Forums. http://www.dxy.com (date last accessed 31 May 2007). 